\title{
Unique Contrast Patterns from Resonance Enhanced Chiral SHG of Cell Membranes
}

\author{
Ping Yan, Andrew C. Millard, Meide Wei, and Leslie M. Loew \\ Center for Cell Analysis and Modeling, University of Connecticut Health Center, Farmington, \\ Connecticut 06030,E-mail: les@volt.uchc.edu
}

\begin{abstract}
Chirality can produce novel non-linear optical effects that may form the basis for new imaging contrast agents. In this paper we developed a new chiral chromophore $\mathbf{2}$, which is the dimer of a known voltage sensitive dye, monomer $\mathbf{1}$, with the chirality originating from the twisted orientation between two subunits. Racemic dimer and monomer $\mathbf{1}$ were used as the references to study the effect of chirality in SHG microscopy of live cells. All these dyes selectively stain the outer leaflets of cell membranes, producing strong resonance-enhanced SHG images. At the symmetric junction between two adherent cells, monomer or racemic dimer SHG is forbidden due to centrosymmetry and indeed little SHG was observed ( $10 \pm 1 \%$ relative to nonjunction). When stained with the chiral dimer the junction is no longer centrosymmetric and much stronger SHG was observed $(39 \pm 4 \%$ relative to nonjunction). Plane polarized light produces highly polarized images of spherical cells stained with racemic dye, but for the chiral dye the polarized pattern is largely eliminated by the chiral SHG emanating from the subresolution membrane convolutions.
\end{abstract}

Second harmonic generation (SHG) is a nonlinear optical process in which two input photons generate one coherent output photon with twice the frequency. It is forbidden where there is an inversion symmetry and this constraint makes it a sensitive tool for the study of interfaces and surfaces. ${ }^{1,2}$ Special attention has been paid to the nonlinear effect of chiral molecules due to the fact that they are ubiquitous in biological systems and unable to form structures of inversion symmetry. Pioneering works on SHG from chiral surfaces and thin films, ${ }^{3}$ sum frequency generation (SFG) from chiral solutions, ${ }^{4}$ and SHG imaging of chiral surfaces ${ }^{5}$ are beginning to establish a field of chiral-specific nonlinear spectroscopy and microscopy. ${ }^{6}$

Our laboratory has been exploring SHG as a new imaging contrast mechanism for cell structure and function. ${ }^{7}$ The hemicyanine dye $\mathbf{1}$, which we have called di-4-ANEPPDHQ, ${ }^{8}$ is a typical dye designed as a probe for transmembrane electric potential because it displays a molecular Stark effect. ${ }^{8-10}$ The underlying change in electron distribution between the ground and excited state is also a key prerequisite for resonance-enhanced SHG. ${ }^{11}$ When the excitation wavelength is close to twice the absorption peak, resonance enhancements of the SHG by an order of magnitude are typical for arrays of such dyes bound to one leaflet of the lipid bilayer in the plasma membrane of a cell. ${ }^{12}$ Utilizing the forward and backward light signals respectively, SHG and two-photon fluorescence (2PF) images can be obtained simultaneously on a modified scanning confocal imaging system. ${ }^{13-15}$ These two complementary imaging modalities have proven useful in understanding cell processes involving symmetry changes.

16 In earlier work, we appended chiral side chains to the hemicyanine chromophore and observed further enhancement of the resonance SHG signals. ${ }^{15}$ In this communication, dimer 2 with the chiral center at the chromophore was synthesized. We find unique contrast patterns 
in images of cell membranes stained with chiral 2 compared to a racemic mixture or the achiral monomer 1 .

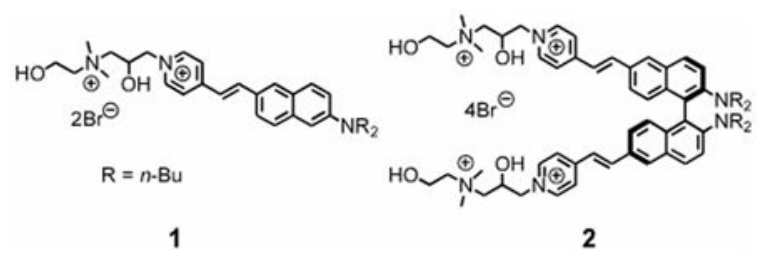

Binaphthyl derivatives have been the most popular model compounds for chiral SHG studies due to their strong chiral response and commercial availability. 3,5,17 As shown in Scheme 1, the synthesis of dimer 2 starts with (R)-1,1'-binaphthyl-2,2'-diamine, which is commercially available in optically pure form (Aldrich). Regiospecific bromination and reductive alkylation were achieved at low temperatures, and then the dibromide $\mathbf{5}$, which is highly resistant to racemization at $110{ }^{\circ} \mathrm{C}$, was coupled to vinyl pyridine and quaternized using the established methods for the monomer. ${ }^{8}$ The CD spectrum of 2 (see Supporting Information) shows a large bisignate band, which is associated with exciton coupling of the long-axis polarized electronic transitions. ${ }^{18}$ For comparison, racemic dimer was also synthesized starting from racemic $1,1^{\prime}$ binaphthyl-2,2'-diamine.

SHG intensity is governed by the second-order nonlinear susceptibility $\chi_{\mathrm{ijk}}$, a third-rank tensor consisting of 27 elements. ${ }^{19}$ A monolayer of achiral molecules belongs to $C_{\infty \mathrm{v}}$ symmetry and the tensor has three independent nonzero elements $\left(\chi_{1}=\chi_{\mathrm{zzz}}, \chi_{2}=\chi_{\mathrm{zxx}}=\chi_{\mathrm{zyy}}, \chi_{3}=\chi_{\mathrm{xzx}}=\right.$

$\chi_{\mathrm{xxz}}=\chi_{\mathrm{yzy}}=\chi_{\mathrm{yyz}}$; ; however, for chiral molecules the monolayer belongs to $\mathrm{C}_{\infty}$ symmetry and there is a fourth chiral element $\left(\chi_{4}=\chi_{x y z}=\chi_{x z y}=-\chi_{y z x}=-\chi_{y x z}\right) .{ }^{19}$ The chiral SHG element can be separated and imaged by employing a special counter-propagating optical setup. ${ }^{5}$

However, a geometry that is commonly observed in cell cultures provides ready detection of chiral SHG. In a situation with two monolayers facing each other, as in the region of apposing membranes between adherent cells, all the achiral components cancel in the $\mathrm{D}_{\infty \mathrm{h}}$ symmetry. 20 Therefore, our nonlinear microscope can be used to directly image the remaining chiral SHG in such regions. Since the broad absorption bands for the monomer and the dimers have maxima at $473 \mathrm{~nm}$ and $449 \mathrm{~nm}$, respectively, we used a Ti:sapphire laser tuned to $916 \mathrm{~nm}$ to effect resonance enhancement. Figure 1 shows SHG images (top panel) and 2PF images (bottom panel) of N1E-115 neuroblastoma cells stained with monomer, racemic dimer, and chiral dimer, respectively from left to right. All three dyes can align well on the outer leaflets of cell membranes by virtue of their amphiphilic structures and produce strong SHG signals. The difference is rather subtle when looking at isolated single cells but the effect of chirality manifests itself in the junction regions between two adherent cells: achiral SHG elements from two apposing cell membranes cancel while chiral elements interfere constructively. As expected there is little SHG signal from the junction parts of the cells stained with the monomer or racemic dimer (Figure 1a and 1b) while substantial signals can be observed for the chiral dimer (Figure 1c). 2PF is a non-coherent process and the signals from the junction parts are strong for all cases (Figure 1d, 1e, and 1f), indicating that these regions have ample dye densities that are comparable for all 3 dyes. For quantitative comparison the SHG signals need to be normalized by the corresponding $2 \mathrm{PF}$ signals to account for the non-uniform staining. The ratio of junction/non-junction SHG (normalized by $2 \mathrm{PF}$ ) proves to be a reliable indicator of chirality: it is $0.10 \pm 0.01$ for the monomer $(\mathrm{N}=11)$ and racemic dimer $(\mathrm{N}=16)$, compared with 0.39 \pm 0.04 for the chiral dimer $(\mathrm{N}=28)$. Thus, resonance enhanced chiral SHG expands the scope of SHG imaging to centrosymmetric structures. 
The images in Figure 1 were obtained with circularly polarized incident light to avoid any asymmetries due to anisotropic cell geometry. We then explicitly investigated the contrast patterns that could be obtained with plane polarized light using the lymphocyte cell line L1210. These are small spherical ( $\sim 10 \mu \mathrm{m}$ in diameter) cells with a convoluted membrane at the ultrastructural level, where the convolutions are at a distance scale shorter than the optical coherence length. Figure 2 shows SHG images from L1210 cells stained with either racemic or chiral 2 and imaged with either linearly polarized or circularly polarized light. The dependence of SHG on angle is manifest as arcs of intensity in the case of the racemic dye imaged with linearly polarized light. This corresponds to a preference for cell surface regions perpendicular to the electric field vector of the light, with the membrane convolutions effectively averaged out. There is no such clear spatial variation for the chiral dye and little difference between linear and circular polarization. This was the consistent pattern for all of several dozen cells examined. Thus, SHG is observed at all angles because the chiral component, $\chi_{4}$, is not annihilated by the convoluted membrane. We see similar contrast patterns in the case of fluorescence, which also depends on angle but not on symmetry: sharp intensity arcs are often barely perceptible in cell membranes (see Supporting Information).

We have developed a new chiral chromophore that provides unique contrast patterns in resonance enhanced SHG from stained cell membranes. Chiral SHG allows imaging of $\mathrm{D}_{\infty \mathrm{h}}$ structures, which are not visible with traditional SHG. At the same time chiral SHG possesses high selectivity: unlike fluorescence, it is still forbidden in isotropic solutions. ${ }^{20}$ Biological structures, such as lipid bilayers, microfilaments and microtubules, which are difficult or impossible to label non-centrosymmetrically, offer a rich basis for the application of chiral SHG. Indeed, chiral SHG fills a contrast niche among non-linear optical probes: achiral SHG is specific for asymmetric interfaces; chiral SHG visualizes symmetric interfaces but is forbidden in isotropic solutions; ${ }^{20}$ chiral SFG is allowed in chiral solutions; ${ }^{4} 2 \mathrm{PF}$ is, of course, not subject to any symmetry constraints. Conversely, the variety of geometries offered by biological structures provides a rich basis for photophysical investigation of novel molecular arrays.

\section{Supplementary Material}

Refer to Web version on PubMed Central for supplementary material.

\section{Acknowledgements}

This study was supported by the National Institutes of Health via grants No. EB001963 and U54RR022232.

\section{References}

1. Shen YR. Nature 1989;337:519.

2. Eisenthal KB. Chem Rev 1996;96:1343. [PubMed: 11848793]

3. a Hicks JM, Petralli-Mallow T. Appl Phys B 1999;68:589. b Verbiest T, Kauranen M, Persoons A. J Mater Chem 1999;9:2005.

4. Ji N, Zhang K, Yang H, Shen YR. J Am Chem Soc 2006;128:3482. [PubMed: 16536497]

5. Kriech MA, Conboy JC. J Am Chem Soc 2005;127:2834. [PubMed: 15740102]

6. Simpson GJ. ChemPhysChem 2004;5:1301. [PubMed: 15499846]

7. Campagnola PJ, Loew LM. Nat Biotechnol 2003;21:1356. [PubMed: 14595363]

8. Obaid AL, Loew LM, Wuskell JP, Salzberg BM. J Neurosci Methods 2004;134:179. [PubMed: 15003384]

9. Loew LM, Bonneville GW, Surow J. Biochemistry 1978;17:4065. [PubMed: 708694]

10. Loew LM, Scully S, Simpson L, Waggoner AS. Nature 1979;281:497. [PubMed: 492309]

11. Dirk CW, Twieg RJ, Wagniere G. J Am Chem Soc 1986;108:5387. 
12. Williams DJ. Angew Chem, Int Ed Engl 1984;23:690.

13. Millard, AC.; Campagnola, PJ.; Mohler, W.; Lewis, A.; Loew, LM. Meth Enzymol. Marriott, G.; Parker, I., editors. 361B. Academic Press; San Diego: 2003. p. 47-69.

14. Campagnola PJ, Clark HA, Mohler WA, Lewis A, Loew LM. J Biomed Opt 2001;6:277. [PubMed: 11516317]

15. Campagnola PJ, Wei MD, Lewis A, Loew LM. Biophys J 1999;77:3341. [PubMed: 10585956]

16. Millard AC, Terasaki M, Loew LM. Biophys J 2005;88:L46. [PubMed: 15849238]

17. Deussen HJ, Hendrickx E, Boutton C, Krog D, Clays K, Bechgaard K, Persoons A, Bjornholm T. J Am Chem Soc 1996;118:6841.

18. Berova, N.; Nakanishi, K. Circular Dichroism: Principles and Applications. Berova, N.; Nakanishi, K.; Woody, RW., editors. Wiley-VCH; New York: 2000. p. 337-382.

19. Byers JD, Yee HI, Petrallimallow T, Hicks JM. Phys Rev B 1994;49:14643.

20. Giordmaine JA. Phys Rev 1965;138:A1599. 

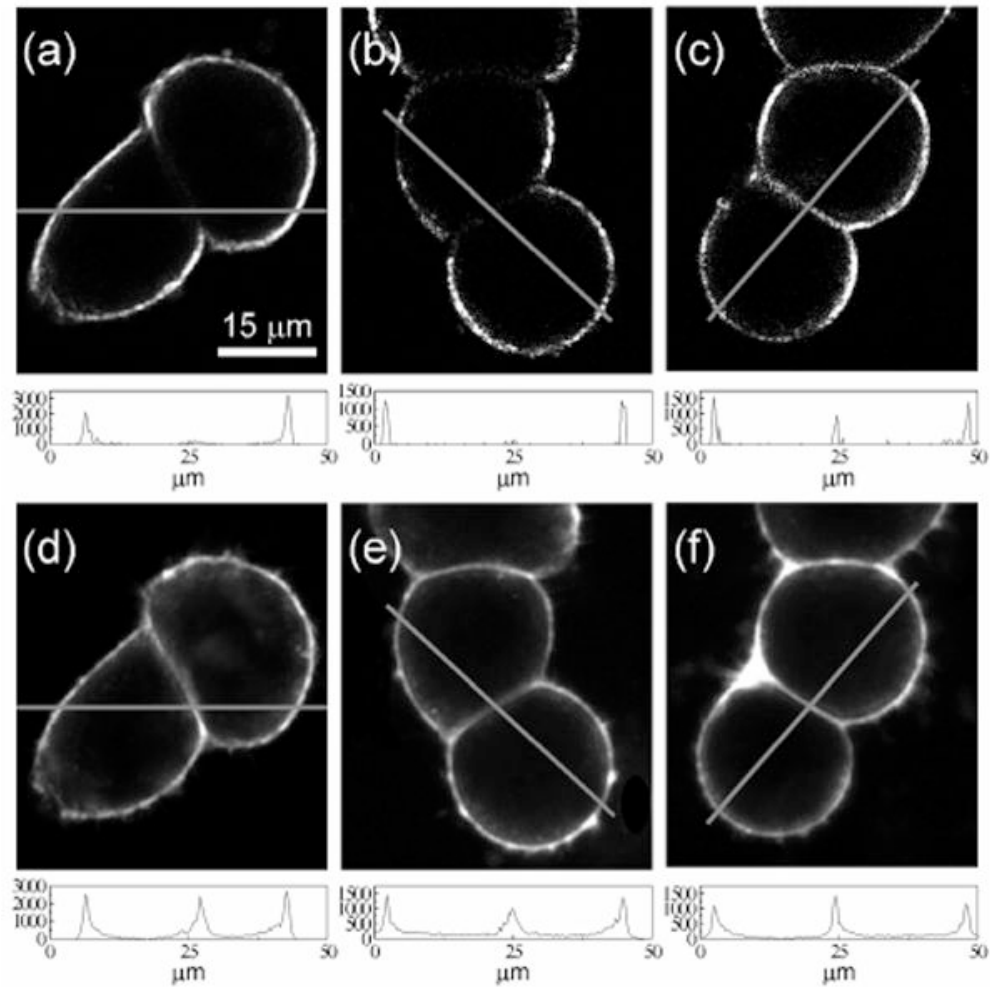

Figure 1.

Effect of chirality on SHG imaging. (a, b, c) SHG and (d, e, f) 2PF images for cells stained with monomer (a, d), racemic dimer (b, e), or chiral dimer (c, f). Line scans through the junctions are shown at the bottom of each image. The samples were excited with circularly polarized light at a power level of $\sim 14 \mathrm{~mW}$ for the monomer and $\sim 28 \mathrm{~mW}$ for the dimers. Each image is a Kalman average of three acquisitions, corresponding to $\sim 8 \mathrm{~s}$ total acquisition time. 

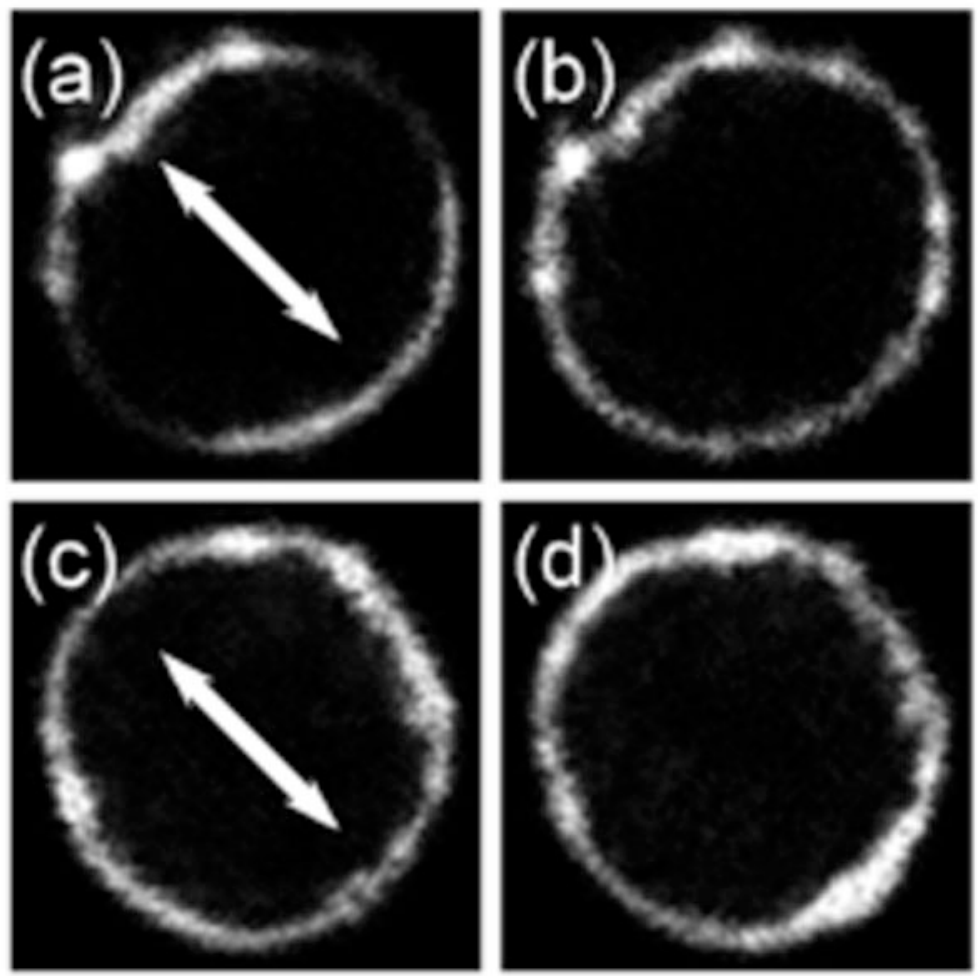

Figure 2.

Effect of polarization on SHG imaging. (a) and (b) show one L1210 cell stained with racemic dimer; (c) and (d) show another cell stained with chiral dimer. (a) and (c) were imaged using linearly polarized light, as shown by the arrow; (b) and (d) were imaged using circularly polarized light. 


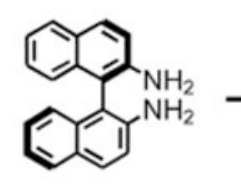

3

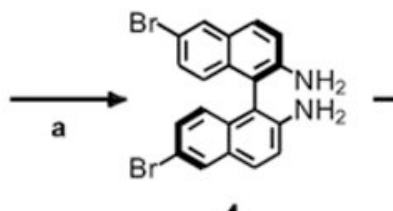

4
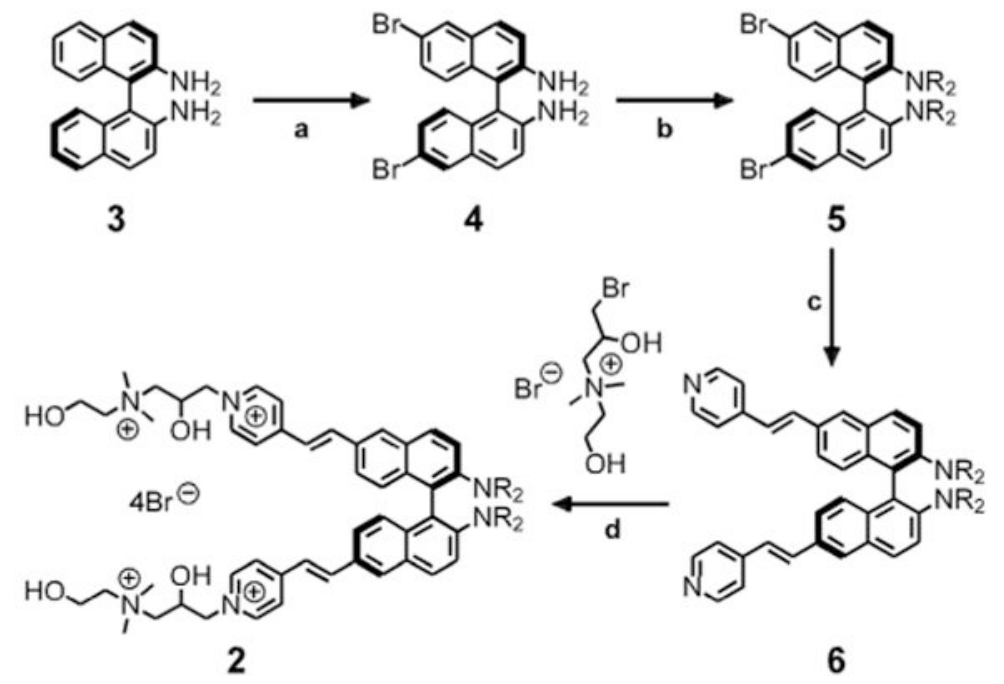

6

Scheme 1a.

${ }^{a} \mathrm{R}=n$-Bu, conditions: (a) $\mathrm{Br}_{2},-20{ }^{\circ} \mathrm{C}$; (b) $\mathrm{C}_{3} \mathrm{H}_{7} \mathrm{CHO} / \mathrm{H}_{2} \mathrm{SO}_{4}, \mathrm{NaBH}(\mathrm{OAc})_{3}$, RT; (c) $\mathrm{Pd}$ $(\mathrm{OAc})_{2}, \mathrm{NEt}_{3}, 110^{\circ} \mathrm{C}$; and (d) DMF, $110^{\circ} \mathrm{C}$. 